\title{
In Vivo Deletion of Immunoglobulin Domains 5 and 6 in Neurofascin (Nfasc) Reveals Domain-Specific Requirements in Myelinated Axons
}

\author{
Courtney Thaxton, ${ }^{1}$ Anilkumar M. Pillai, ${ }^{1}$ Alaine L. Pribisko, ${ }^{1}$ Marilyne Labasque, ${ }^{2}$ Jeffrey L. Dupree, ${ }^{3}$ \\ Catherine Faivre-Sarrailh, ${ }^{2}$ and Manzoor A. Bhat ${ }^{1}$ \\ ${ }^{1}$ Department of Cell and Molecular Physiology, and UNC-Neuroscience Center, University of North Carolina School of Medicine, Chapel Hill, North Carolina \\ 27599-7545, 2 Centre de Recherche en Neurobiologie et Neurophysiologie de Marseille, UMR 6231 CNRS, Université de la Méditerranée Aix-Marseille II, \\ Marseille 13344, France, and ${ }^{3}$ Department of Anatomy and Neurobiology, Virginia Commonwealth University, Richmond, Virginia 23298-0709
}

The formation of paranodal axo-glial junctions is critical for the rapid and efficient propagation of nerve impulses. Genetic ablation of genes encoding the critical paranodal proteins Caspr, contactin (Cont), and the myelinating glia-specific isoform of Neurofascin $\left(\mathrm{Nfasc}^{\mathrm{NF155}}\right.$ ) results in the disruption of the paranodal axo-glial junctions, loss of ion channel segregation, and impaired nerve conduction, but the mechanisms regulating their interactions remain elusive. Here, we report that loss of immunoglobulin (Ig) domains 5 and 6 in Nfasc ${ }^{\mathrm{NF} 155}$ in mice phenocopies complete ablation of $\mathrm{Nfasc}^{\mathrm{NF} 155}$. The mutant mice lack paranodal septate junctions, resulting in the diffusion of Caspr and Cont from the paranodes, and redistribution of the juxtaparanodal potassium channels toward the nodes. Although critical for $\mathrm{Nfasc}^{\mathrm{NF} 155}$ function, we find that Ig5-6 are dispensable for nodal $\mathrm{Nfasc}^{\mathrm{NF} 186}$ function. Moreover, in vitro binding assays using Ig5-6 deletion constructs reveal their importance for the association of $\mathrm{Nfasc}^{\mathrm{NF} 155}$ with Cont. These findings provide the first molecular evidence demonstrating domain-specific requirements controlling the association of the paranodal tripartite complex in vivo. Our studies further emphasize that in vivo structure/function analysis is necessary to define the unique protein-protein interactions that differentially regulate the functions of Neurofascins during axonal domain organization.

\section{Introduction}

The organization of ion channels within specific subcellular domains along myelinated axons is essential for proper nerve conduction. Specialized axo-glial septate-like junctions, formed between the glial paranodal loops and the underlying axolemma, mediate the segregation of ion channels within myelinated axons (Salzer, 2003; Thaxton and Bhat, 2009). Although numerous proteins reside within the paranodes, to date only three proteins have been identified as integral components of the paranodal septate junctions: contactin (Cont) and Caspr in the axon, and the 155 $\mathrm{kDa}$ isoform of Neurofascin ( $\mathrm{Nfasc}^{\mathrm{NF} 155}$ ) expressed specifically in myelinating glia (Menegoz et al., 1997; Bhat et al., 2001; Boyle et al., 2001; Sherman et al., 2005; Zonta et al., 2008; Pillai et al., 2009). Genetic ablation of the genes encoding these proteins in mice results in loss of axo-glial junctions, ion channel disorganization, impaired nerve conduction, and ultimately axonal degeneration (Bhat et al., 2001; Boyle et al., 2001; Pillai et al., 2009).

Received Dec. 1, 2009; revised Jan. 28, 2010; accepted Feb. 19, 2010.

This work was supported by National Institutes of Health Grants NS050356 and GM063074 (to M.A.B.) and National Multiple Sclerosis Society grants (to M.A.B. and C.F.S.). We are grateful to Dr. Klaus-Armin Nave (Max Planck Institute of Experimental Medicine, Germany) for generously sharing the (np-Cre mouse strain. We thank Dr. E. Perl for his generous support and advice with electrophysiology. We also thank Dr. A. Fanning and our fellow laboratory members for critical discussions and comments.

Correspondence should be addressed to Manzoor A. Bhat, Department of Cell and Molecular Physiology, UNCNeuroscience Research Center, University of North Carolina School of Medicine, Chapel Hill, NC 27599-7545. E-mail: manzoor_bhat@med.unc.edu.

DOI:10.1523/JNEUROSCI.5951-09.2010

Copyright $\odot 2010$ the authors $\quad 0270-6474 / 10 / 304868-09 \$ 15.00 / 0$
Although characterized for their requirement in paranodal organization, the mechanisms regulating the potential interactions between Cont, Caspr, and Nfasc ${ }^{\mathrm{NF} 155}$ remain unclear.

Myelinating glia-specific ablation of $\mathrm{Nfasc}^{\mathrm{NF155}}$ in a spatiotemporal manner resulted in axonal domain disorganization, including the diffusion of Caspr and Cont from paranodes, and instability of the paranodal region (Pillai et al., 2009). Similarly, ablation of Cont and Caspr in mice resulted in the respective disruption of Caspr and $\mathrm{Nfasc}^{\mathrm{NF} 155}$, and Cont and $\mathrm{Nfasc}^{\mathrm{NF} 155}$, from paranodal axo-glial junctions, suggesting that these proteins may interact to coordinate and organize paranodes (Bhat et al., 2001; Boyle et al., 2001). Indeed, several studies have revealed that the localization and stabilization of both Caspr and Cont to the cell membrane is interdependent (Charles et al., 2002; Gollan et al., 2003; Bonnon et al., 2007). Specific in vitro studies have demonstrated a direct association between $\mathrm{Nfasc}{ }^{\mathrm{NF} 155}$ and Cont (Gollan et al., 2003; Bonnon et al., 2007), but the true associative relationship between Nfasc ${ }^{\mathrm{NF} 155}$, Cont, and Caspr, and how they coordinate axo-glial interactions remains unclear.

Here we report that in vivo deletion of immunoglobulin (Ig) domains 5 and 6 in $\mathrm{Nfasc}^{\mathrm{NF} 155}$ mimics its complete ablation and results in the loss of axo-glial junctions and the failure to segregate ion channels within myelinated axons. Interestingly, deletion of Ig5-6 from the neuron-specific Nfasc ${ }^{\mathrm{NF} 186}$ had no effect on its localization or the overall organization of the nodes, revealing isoform-specific requirements for Ig5-6 within Neurofascins. Moreover, in vitro binding assays indicate that Ig5-6 are essential 
for $\mathrm{Nfasc}^{\mathrm{NF} 155}$ interactions with Cont but are dispensable for Nfasc ${ }^{\mathrm{NF} 186}$ function. Together, our results suggest that Nfasc isoforms use distinct protein-protein interaction modules to organize and stabilize specific axonal domains in myelinated axons.

\section{Materials and Methods}

Animals. All animal procedures were carried out under University of North Carolina Institutional Animal Care and Use Committee-approved guidelines. The Cnp-Cre mice were provided by Dr. Klaus Nave (Max Planck Institute, Germany). $\beta$-Actin-Cre mice were obtained from the Jackson Labs.

Conditional targeting of Nfasc gene. The strategy to delete the genomic region encoding Ig domains 5 and 6 in the $N f a s c$ gene was similar to that described previously (Pillai et al., 2009). To conditionally ablate Ig5-6 in $\mathrm{Nfasc}$ locus, a targeting construct was generated in which loxP sites were targeted to flank exons 11 and 15 of Nfasc. A bacterial artificial chromosome (BAC) clone (bMQ228D3) from 129 AB2.2 BAC library was obtained from the Sanger Center, UK, which contained the targeted Nfasc exons. BAC recombineering technology was applied, and a polymerase II promoter directed Diphtheria toxin (DT) vector containing short PCRgenerated arms homologous to that of Nfasc DNA sequence was transformed into E. coli DY380 recombinant bacterial strain (heat induced RED and GAM recombinase complex) containing the Nfasc BAC. Using this approach, a $\sim 9.4 \mathrm{~kb}$ fragment of $N f a s c$ BAC was retrieved into the $D T$ vector by means of gap repair. The first loxP site was inserted into intron 10 and another loxP-neo-loxP cassette in intron 15 . Confirmation of the functionality of the loxP sites and the proper insertion of these sites was analyzed by restriction enzyme digestion before embryonic stem (ES) cell targeting. The construct was then linearized and electroporated into ES cells, followed by screening of the targeted cells by PCR amplification and standard molecular biological methods for positive clones as previously described (Bhat et al., 2001).

Plasmids. The extracellular domain-gliomedin-Fc construct was from Dr. E. Peles (Weizmann Institute, Rehovot, Israel), transmembrane-gliomedin construct (pcDNA3/gliomedin-TM) was from Dr. J. Devaux (CRN2M, France), and HA-tagged NF186 (pEGFP-C1/HA-NF186) was from Dr. V. Bennett (Duke University, Durham, NC). NF155-Fc and NF186 deleted for

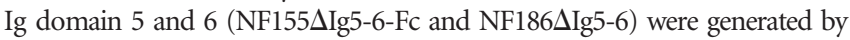
QuickChange mutagenesis (Stratagene). The plasmids encoding NF155-Fc fusion (pIgplus-NF155), F3/Contactin (pRc-CMV-F3), and Caspr (pBK-CMVpnd) have been described previously (Bonnon et al., 2007; Charles et al., 2002; Durbec et al., 1994). The primers used for creating Ig5-6 deletions were: 5'-ATGCCTTCGTCAGCGTATTACGGCCAGACC-3', forward primer, and 5' ${ }^{\prime}$-TAATACGCTGACGAAGGCATTGGCCAGCAGGTAGCCATG-3', reverse primer. All mutant constructs were verified by sequencing Cogenics).

Antibodies and reagents. The following antisera were previously described: guinea pig and rabbit anti-Caspr (Bhat et al., 2001), guinea pig anti-NF186, and rat anti-pan Neurofascin (NFct) (Pillai et al., 2009). The rabbit anti-Caspr2 and rat anti-Ankyrin $\mathrm{G}$ (AnkG) antibodies were generated for this study. Other primary antibodies used include: mouse anti-potassium channel $\left(\mathrm{K}_{\mathrm{v}} 1.1\right.$; Sigma), mouse anti-pan sodium channel $\left(\mathrm{Na}_{\mathrm{v}}\right.$ pan; Sigma), rabbit anti-ezrin binding protein 50 (EBP50; Abcam), rabbit anti- neuronal cell adhesion molecule (NrCAM; Abcam), rabbit anti-Gliomedin (Glmdn; Abcam), rabbit anti-Calbindin (CalB; Sigma), rat anti-HA (Roche), rabbit anti-human Fc (Sigma), and mouse antiHuman Fc (Sigma). Rabbit anti-contactin used for immunoblotting was generously provided by Dr. James Salzer (New York University). Additionally, for cell culture experiments, polyclonal antisera specific for F3/ Contactin (pAbF3) was used as described elsewhere (Rigato et al., 2002). The rabbit anti-Gliomedin (Ab7202) was generously provided by Dr. E. Peles. Secondary antibodies used for immunofluorescence include Alexa Fluor conjugated $-488,-568$, and -647 (Invitrogen), and goat antiHuman IgG (Fc specific)-TRITC (Sigma). The horseradish peroxidase (HRP)-conjugated secondary antibodies used were purchased from Jackson Immunologicals.

Tissue preparation, immunostaining, and immunoblotting. All tissues preparation was carried out as previously described (Bhat et al., 2001;
Pillai et al., 2009). Briefly, sciatic nerves were harvested from anesthetized mice, followed by fixation in $4 \%$ paraformaldehyde (in PBS) for 15 to $30 \mathrm{~min}$ at $4^{\circ} \mathrm{C}$ with rocking. The nerves were washed with PBS several times and either stored at $4^{\circ} \mathrm{C}$ or directly teased onto slides. The slides were dried overnight at room temperature (RT) followed by immunostaining, or stored at $-80^{\circ} \mathrm{C}$ until processed. For the preparation of spinal cord and cerebellar tissues, mice were anesthetized and transcardially perfused with ice-cold $4 \%$ paraformaldehyde (in PBS). The tissues of interest were dissected out and post-fixed in $4 \%$ paraformaldehyde overnight at $4^{\circ} \mathrm{C}$. The tissues were then rinsed several times in PBS and sliced to a thickness of $30 \mu \mathrm{m}$ using a Vibratome (Leica). The sliced sections were then immediately immunostained as described previously (Pillai et al., 2009). All images from the processed tissues were acquired under identical settings using a Bio-Rad Radiance 2000 confocal microscope. The images were processed in Adobe Photoshop using identical settings between control and mutant images. Immunoblotting of protein lysates was carried out as previously described (Pillai et al., 2009).

Immunoblotting. Spinal cords from age-matched wild-type and mutant mice were harvested and either directly processed or frozen at $-80^{\circ} \mathrm{C}$ until further use. Spinal cords were suspended in ice cold homogenization buffer ( $50 \mathrm{~mm}$ Tris- $\mathrm{HCl}, \mathrm{pH} 7.5,150 \mathrm{~mm} \mathrm{NaCl}, 320 \mathrm{~mm}$ sucrose, 10 mm EDTA, pH 8.0, and a protease inhibitor cocktail tablet). The spinal cords were homogenized on ice by 25 to 50 stokes using a glass mortar and pestle. The resulting homogenate was centrifuged at $1000 \times$ $g$ for $10 \mathrm{~min}$ at $4^{\circ} \mathrm{C}$. The supernatant was removed and subjected to further centrifugation at $13,000 \mathrm{rpm}$ for $20 \mathrm{~min}$ at $4^{\circ} \mathrm{C}$. The resulting pellet was resuspended in extraction buffer ( $50 \mathrm{~mm}$ Tris- $\mathrm{HCl}$, pH 7.5, 150 mм NaCl, 10 mм EDTA, pH 8.0, 1\% Triton X-100, and a protease inhibitor cocktail tablet) and incubated for 10 to $30 \mathrm{~min}$ at $4^{\circ} \mathrm{C}$ with gentle rocking. The extraction was then centrifuged at 13,000 rpm for $20 \mathrm{~min}$ at $4^{\circ} \mathrm{C}$ and the supernatant collected. The lysate was subjected to a Lowry Assay (BC assay, Bio-Rad) to determine protein concentration, and equal amounts were resolved on SDS-PAGE. The proteins were transferred onto nitrocellulose membranes, followed by blocking with $5 \%$ milk [dissolved in PBS with $0.1 \%$ Tween-20 (PBS-T) ]. The membranes were incubated with primary antibodies for $1 \mathrm{~h}$ at RT or overnight at $4^{\circ} \mathrm{C}$ and were subsequently washed in PBS-T and incubated with horseradish peroxidase (HRP)-conjugated secondary antibodies for $40 \mathrm{~min}$ at RT. Signals were determined by the application of chemiluminescent substrate and exposure of membranes to autoradiographic film.

Cell culture and transfection. The $\mathrm{N}$-glycosylation mutant $\mathrm{CHO}$ cell line (CHO-Lec1) (Stanley and Ioffe, 1995) was kindly provided by Dr. E. Fenouillet (Institut Jean Roche). CHO-Lec1 and HEK-293 cells were grown in Dulbecco's modified eagle medium (DMEM) supplemented with $10 \%$ fetal calf serum (FCS; Invitrogen) were transiently transfected using jetPEI (Ozyme). NF155-Fc, NF155 Ig5-6-Fc, and Gliomedin-Fc were produced in the supernatant of transfected HEK-293 cells and used for binding experiments as previously described (Charles et al., 2002; Lonigro and Devaux, 2009).

Binding of Fc-chimera and immunofluorescence. Cells transfected with Cont, Cont + Caspr, Gliomedin, or NF186 were incubated with NF155Fc, NF155 Ig5/6-Fc, or Gliomedin-Fc $(5 \mu \mathrm{g} / \mathrm{ml})$ for $30 \mathrm{~min}$ at $37^{\circ} \mathrm{C}$, washed, and then incubated with TRITC-conjugated anti-Human Fc before fixation with $4 \%$ paraformaldehyde. For surface immunostaining of HA-tagged constructs, fixed cells were incubated with rat anti-HA antibody followed by fluorescently-labeled secondary antibodies diluted in PBS containing 3\% bovine serum albumin (BSA). After washing in PBS, the cells were mounted in Mowiol (Calbochiem) and examined using an apotome AxioObserver Z1 inverted microscope under the control of Axiovision software (Zeiss).

Protein pull-down assay. CHO-Lec1 cells transiently transfected with Contactin were homogenized on ice with lysis buffer (containing $60 \mathrm{~mm}$ octyl glucoside and $1 \%$ Triton X-100) as previously described (Charles et al., 2002). Solubilized proteins ( $1.5 \mathrm{mg}$ per condition) were incubated with Fc fusion proteins $(100 \mu \mathrm{g})$ immobilized onto protein A-Sepharose CL-4B beads (200 $\mu$ lGE Healthcare). Affinity retained proteins were analyzed by immunoblotting as described previously (Bonnon et al., 2007).

Transmission electron microscopy. Transmission electron microscopy (TEM) of age-matched wild-type Cnp-Cre;Nfasc ${ }^{\text {IgFlox }}, \beta$-Act-Cre;Nfasc ${ }^{\text {Iglox }}$ 
mutant mice was carried out essentially as described previously (Pillai et al., 2009).

Additional experimental procedures are provided in the supplemental Materials and Methods section (available at www.jneurosci.org).

\section{Results}

\section{Targeted deletion of Ig domains 5 and 6 in Neurofascin}

We recently demonstrated that the myelinating glia-specific isoform of Neurofascin (Nfasc ${ }^{\mathrm{NF} 155}$ ) is required for the formation and maintenance of paranodal axo-glial junctions (Pillai et al., 2009). To further dissect the role of $\mathrm{Nfasc}^{\mathrm{NF} 155}$ in paranodal organization, we generated mice specifically lacking Ig domains 5 and 6 (Ig5-6) from $N$ fasc (Fig. $1 A$ ). These two domains represent the midpoint of the extracellular region in both the glial and neuronal isoforms of Nfasc and are precisely encoded by exons 11-15 (http://www.ensembl.org/Mus_musculus). A targeting construct where exons 11 and 15 were flanked by loxP sites (Fig. $1 B$, red arrows) was used to generate $N$ fasc ${ }^{\text {IgFlox }}$ mice. The genotypes of the wild-type $(+/+)$, heterozygous $\left(+/ N f a s c^{I g F l o x}\right)$, and homozygous ( $N$ fasc ${ }^{\text {IgFlox }}$ ) mice were identified by PCR using specific primers (Fig. 1C). Next, we determined whether loss of Ig5-6 from Nfasc ${ }^{\mathrm{NF} 155}$, using Cnp-Cre (Lappe-Siefke et al., 2003), resulted in an analogous phenotype as complete ablation of $\mathrm{Nfasc}^{\mathrm{NF} 155}$. Examination of the Cnp-Cre; Nfasc ${ }^{\text {IgFlox }}$ mice revealed phenotypes consistent with that observed in $\mathrm{Nfasc}^{\mathrm{NF} 155}$-null mice including severe ataxia, motor coordination defects, and progressive weight loss (Fig. $1 \mathrm{D}$ and supplemental Figure 1, available at www.jneurosci.org as supplemental material) (Pillai et al., 2009). To confirm isoform-specific deletion of Ig5-6 in Nfasc ${ }^{\mathrm{NF} 155}$ and $\mathrm{Nfasc}^{\mathrm{NF186}}$, immunoblot analysis was carried out on wildtype $(+/+)$, Nfasc ${ }^{\text {NF155 IIg5-6 }}$ (Cnp-Cre;Nfasc ${ }^{\text {IgFlox }}$, Fig. 1E), and Nfasc $^{\Delta \operatorname{Ig} 5-6}\left(\beta\right.$-Act-Cre;Nfasc ${ }^{\text {IgFlox, }}$ Fig. $\left.1 F\right)$ spinal cords using a pan-Neurofascin (NFct) antibody (Pillai et al., 2009). In wildtype $(+/+)$ spinal cord lysate, nodal $\mathrm{Nfasc}^{\mathrm{NF} 186}$ and glial Nfasc ${ }^{\mathrm{NF} 155}$ were observed at their respective molecular weights. In both Cnp-Cre;Nfasc ${ }^{\text {IgFlox }}$ and $\beta$-Act-Cre;Nfasc ${ }^{\text {IgFlox }}$ lysates, a 135 $\mathrm{kDa}$ band corresponding to loss of Ig5-6 from Nfasc ${ }^{\mathrm{NF} 155}$ was observed (pink asterisks) and indicates that excision of Ig5-6 did not affect translation of the mutant protein. Additionally, a 166 $\mathrm{kDa}$ band corresponding to deletion of Ig5-6 from Nfasc ${ }^{\mathrm{NF} 186}$ was observed in heterozygous $\left(+/ \beta\right.$-Act-Cre; $\left.N f a s c^{\text {IgFlox }}\right)$ and $\beta$-Act-Cre; $N$ fasc ${ }^{\text {IgFlox }}$ lysates compared to wild-type (Fig. $1 F$, pink asterisks). Caspr and Cont showed no changes between wildtype, Cnp-Cre;Nfasc IgFlox, and $\beta$-Act-Cre;Nfasc ${ }^{\text {IgFlox }}$ lysates. Together, these results demonstrate the successful ablation of Ig5-6 in Nfasc and reveal that loss of Ig5-6 in Nfasc ${ }^{\mathrm{NF} 155}$ phenocopies its complete ablation; furthermore, they highlight an essential role for these domains in the organization of paranodes by $\mathrm{Nfasc}^{\mathrm{NF} 155}$.

\section{Ig5-6 of $\mathrm{Nfasc}^{\mathrm{NF155}}$, but not $\mathrm{Nfasc}{ }^{\mathrm{NF186}}$, are essential for axonal domain organization in myelinated axons}

To examine whether loss of Ig5-6 in Nfasc ${ }^{\mathrm{NF} 155}$ effected paranodal formation and axonal domain organization, we immunostained sciatic nerve fibers with antibodies against key juxtaparanodal, paranodal, and nodal molecules (Fig. 2A-H). Immunoreactivity of the pan-Neurofascin (NFct) antibody (green) revealed specific loss of $\mathrm{Nfasc}^{\mathrm{NF} 155}$ from the paranodes in Cnp-Cre;Nfasc ${ }^{\text {IgFlox }}$ nerves compared to wild-type $(+/+)$ nerves, whereas the localization of nodal Nfasc ${ }^{\mathrm{NF} 186}$ (NF186, red) was unchanged (Fig. $2 A, B$ ). Further examination of the nerve fibers revealed the presence of the mutant Nfasc ${ }^{\mathrm{NF} 155}$ protein within Schmidt-Lantermann incisures in Cnp-Cre; $N f a s c^{\text {IgFlox }}$ fibers (Fig.
$2 B^{\prime}$ ), as in wild-type (Fig. $2 A^{\prime}$ ). In addition, NF155 (green) was observed in a small number of paranodes (arrowheads) in CnpCre; Nfasc ${ }^{\text {IgFlox }}$ nerves (Fig. 2D, $\left.D^{\prime}\right)$ as in wild-type (Fig. 2C-C'), suggesting that loss of Ig5-6 did not affect the transport or the ability of Nfasc ${ }^{\mathrm{NF} 155 \Delta \mathrm{Ig} 5-6}$ to localize to the paranodes or within the glial plasma membrane. In contrast, enriched paranodal Caspr (blue) fluorescence was not observed in Cnp-Cre; $N f a s c^{I g}$. Flox fibers, but was rather diffused throughout the internode (Fig. $2 F-\mathrm{F}^{\prime \prime \prime}$ ) compared to wild-type nerves (Fig. $\left.2 E-E^{\prime \prime \prime}\right)$. In addition, juxtaparanodal shaker-type potassium channels $\left(\mathrm{K}_{\mathrm{v}} 1.1\right.$, red) redistributed into the paranodal space in Cnp-Cre; fasc I gFlox fibers and directly abutted the node (NF186, green). Accordingly, juxtaparanodal Caspr2 was also found mislocalized in the paranodes in Cnp-Cre; Nfasc IfFlox nerves compared to wild-type nerves (supplemental Figure 2, available at www.jneurosci.org as supplemental material). Interestingly, even in the absence of proper paranodal formation, as observed in Cnp-Cre; Nfasc IgFlox mutant nerves, both ankyrin $_{\mathrm{G}}\left(\right.$ AnkG, red) and Nfasc ${ }^{\mathrm{NF} 186}$ (NF186, blue) remained localized to nodes (Fig. $\left.2 H-H^{\prime \prime \prime}\right)$. Together, these findings further demonstrate that deletion of Ig5-6 in Nfasc ${ }^{\mathrm{NF} 155}$ phenocopies its complete ablation and suggest that these domains are essential for $\mathrm{Nfasc}{ }^{\mathrm{NF} 155}$ function in organizing the paranodes.

Because of the significant homology between the Ig domains of Nfasc ${ }^{\mathrm{NF} 155}$ and Nfasc ${ }^{\mathrm{NF} 186}$ (Fig. 1A) (Davis et al., 1996), and the importance of Ig5-6 to Nfasc ${ }^{\mathrm{NF} 155}$ function, we sought to determine whether these domains were equally indispensable for $\mathrm{Nfasc}^{\mathrm{NF} 186}$ function and localization at nodes. To ablate Ig5-6 from $\mathrm{Nfasc}{ }^{\mathrm{NF} 186}$, the $N f a s c^{\text {IgFlox }}$ mice were crossed with the ubiquitously expressed Cre line, $\beta$-Actin-Cre $(\beta$-Act-Cre), which resulted in the excision of Ig5-6 from both isoforms of Nfasc (Fig. $1 F)$. To assess axonal domain organization, we immunostained teased sciatic nerve fibers from wild-type $(+/+)$ and Nfasc ${ }^{\Delta \text { Ig5-6 }}$ $\left(\beta-A c t-C r e ; N f a s c^{I g F l o x}\right)$ mice with the indicated antibodies (Fig. $2 I-N)$. As expected, Caspr (green) localization to paranodes was disrupted in $\beta$-Act-Cre; $N$ fasc ${ }^{\text {IgFlox }}$ fibers (Fig. $2 J-J^{\prime \prime \prime}$ ) compared to wild-type $(+/+)$ fibers (Fig. $\left.2 I-I^{\prime \prime \prime}\right)$. In addition, we found that juxtaparanodal $\mathrm{K}_{\mathrm{v}} 1.1$ were mislocalized in $\beta$-Act-Cre; $N f a s c^{\text {IgFlox }}$ mice, as in Cnp-Cre;Nfasc ${ }^{\text {IgFlox }}$, consistent with loss of Ig5-6 from Nfasc ${ }^{\mathrm{NF155}}$ (supplemental Figure 2, available at www. jneurosci.org as supplemental material). Interestingly, voltage-gated sodium channels ( $\mathrm{Na}_{\mathrm{v}}$ pan, red) remained enriched at nodes and colocalized with NF186 (blue) in $\beta$-Act-Cre;Nfasc ${ }^{\text {IgFlox }}$ nerves as in wild-type. AnkG localization to nodes was also unaffected in $\beta$-Act-Cre; $\mathrm{Nfasc}^{\text {IgFlox }}$ (supplemental Figure 2, available at www.jneurosci.org as supplemental material). These findings suggest that loss of Ig5-6 does not disrupt the ability of Nfasc ${ }^{\mathrm{NF} 186}$ to associate with, or organize other, axonallyexpressed nodal components.

In the peripheral nervous system (PNS), the Schwann cell microvilli overlie the node and express gliomedin (Glmdn), ezrin binding phosphoprotein 50 (EBP50), and phosphorylated ezrin/ radixin/moesin (ERM) proteins (Eshed et al., 2005; MelendezVasquez et al., 2001). Because of their importance in PNS nodal development, we sought to determine whether loss of Ig5-6 in $\mathrm{Nfasc}^{\mathrm{NF} 186}$ affected the expression and/or localization of EBP50 and Glmdn by immunostaining (Fig. $2 \mathrm{~K}-\mathrm{N}$ ). In both wild-type and $\beta$-Act-Cre; Nfasc ${ }^{\text {IgFlox }}$ myelinated fibers, EBP50 (green) colocalized with $\mathrm{Nfasc}^{\mathrm{NF} 186}$ (NFct, blue) at nodes (Fig. $2 K-L$ ). Accordingly, Glmdn (green) remained localized at nodes in $\beta$-ActCre; Nfasc ${ }^{\text {IgFlox }}$ nerves, as in wild-type, where it also colocalized with Nfasc ${ }^{\mathrm{NF} 186}$ (blue, NFct) (Fig. $2 N-N^{\prime \prime \prime}$ ). These results suggest that Ig5-6 of Nfasc ${ }^{\mathrm{NF} 186}$ are not responsible for the associa- 

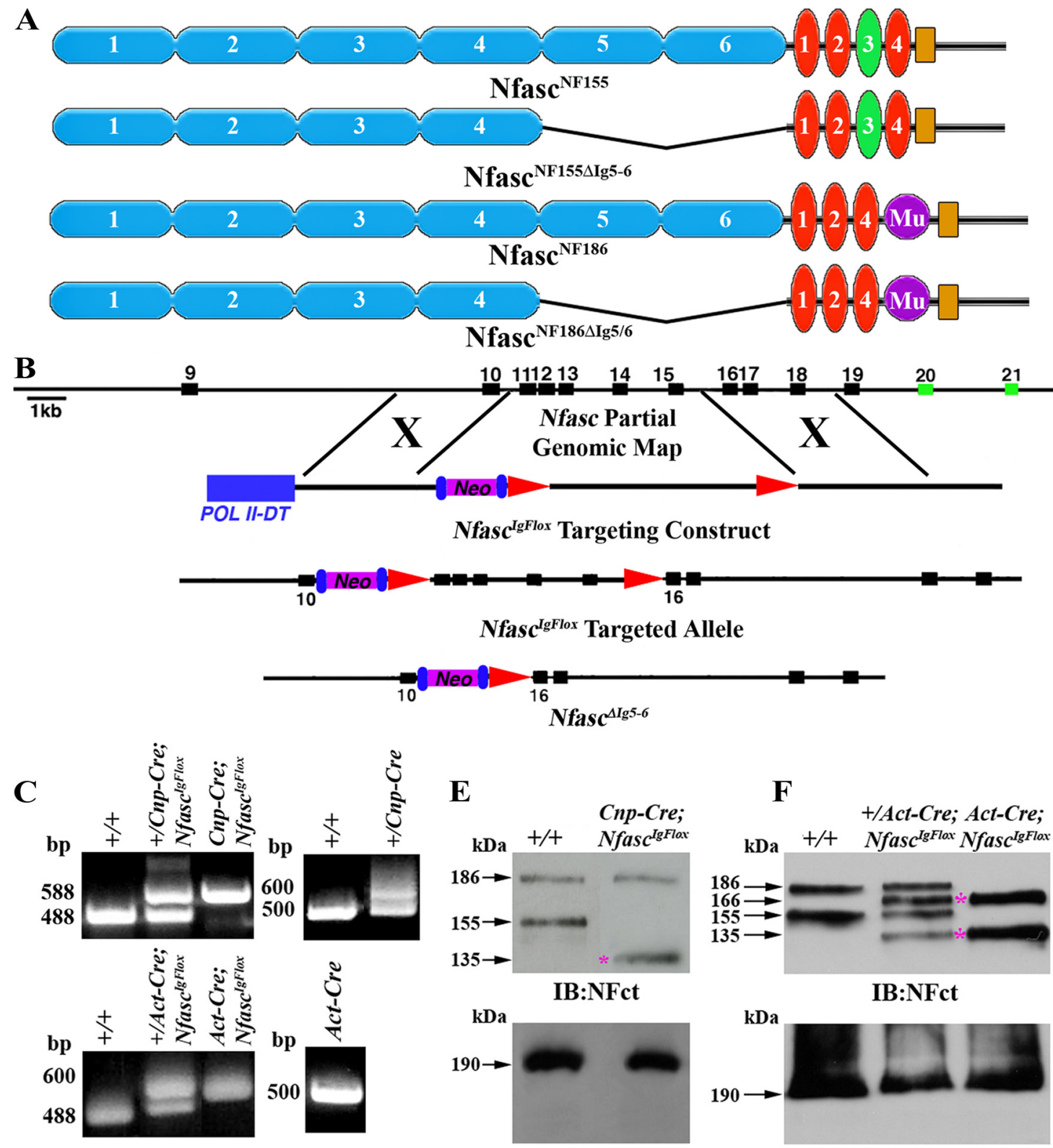

$\mathbf{E}$
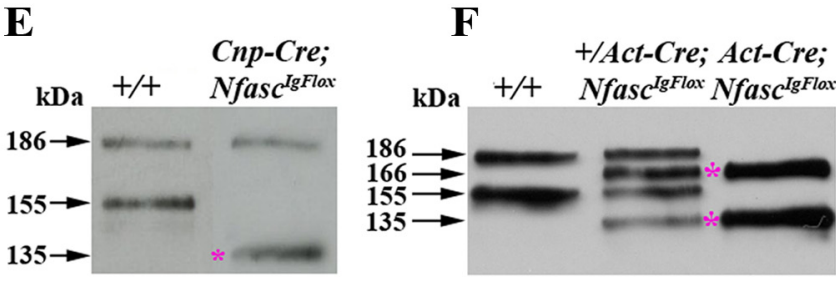

IB:NFct
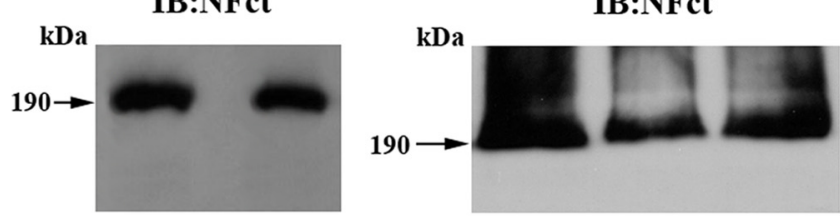

D

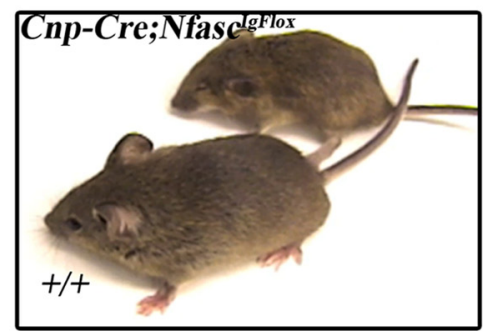

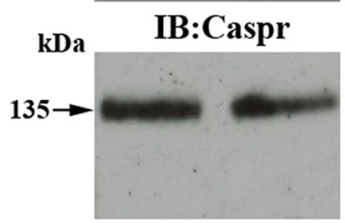

IB:Cont

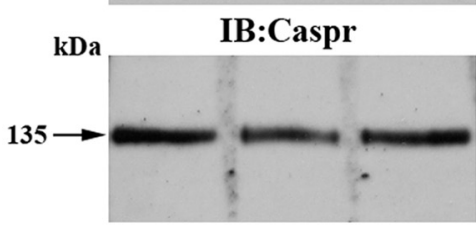

IB:Cont

Figure 1. Genetic ablation of Ig5-6 from Nfasc. A, Schematic diagram illustrating Nfasc wild-type and Nfasc ${ }^{\Delta l g 5-6}$ isoforms. B, Generation of the conditional Nfas ${ }^{\text {dgFlox }}$ and Nfasc ${ }^{\Delta / g 5-6}$ alleles. C, PCR amplification of genomic DNA for identifying wild-type $(+/+)$, heterozygous (+/NfasdgFlox), and homozygous floxed (Nfasc ${ }^{\text {dgFlox }}$ ) Nfasc alleles, and Cre, in (np-Cre;NfasclgFlox and $\beta$-Act-Cre; Nfasc ${ }^{\text {IgFlox }}$ mutant mice. D, Photographic display of the wild-type $(+/+)$ and Nfasc ${ }^{\mathrm{NF} 155 \Delta \operatorname{sg} 5-6}$ (Cnp-Cre; Nfasc $\left.{ }^{\text {gflox }}\right)$ mice. E, Immunoblot analysis of wild-type $(+/+)$ and Nfasc ${ }^{\mathrm{NF} 155 \Delta \lg 55-6}$ (Cnp-Cre; Nfasc ${ }^{\text {IgFlox }}$ ) spinal cord lysates against the antibodies indicated. $\boldsymbol{F}$, Immunoblot analysis of wild-type $(+/+)$, heterozygous $\left(+/ \beta\right.$-Act-Cre; Nfasc $\left.{ }^{\text {lgFlox }}\right)$, and Nfasc ${ }^{\Delta l g 5-6}(\beta$-Act-Cre; Nfasc ${ }^{\text {IgFlox }}$ ) spinal cord lysates against the indicated antibodies. The asterisks denote lg5-6-specific deleted isoforms.

tion, organization, or stabilization of the microvilli or its related proteins to the node.

We further analyzed axonal domain organization in CNS myelinated fibers of wild-type $(+/+)$ and $\mathrm{Cnp}$-Cre; $\mathrm{Nfasc}^{\text {IgFlox }}$ mice (Fig. 2O-R). As in the PNS, juxtaparanodal $\mathrm{K}_{\mathrm{v}} 1.1$ (red) channels were mislocalized, and Caspr (blue) was diffused in Cnp-Cre;
$N f a s c^{I g F l o x}$ white matter (Fig. $2 P-P^{\prime \prime \prime}$ ), whereas NF186 (green) was concentrated at the nodes. In addition, AnkG (red) was properly localized at CNS nodes in Cnp-Cre; $\mathrm{Nfasc}^{\text {IgFlox }}$ spinal cords, where it colocalized with NF186 (blue) regardless of paranodal Caspr (green) (Fig. 2R). Similar results were observed in the CNS of $\beta$-Act-Cre; Nfasc ${ }^{I g F l o x}$ mice (supplemental Figure 2, available at 


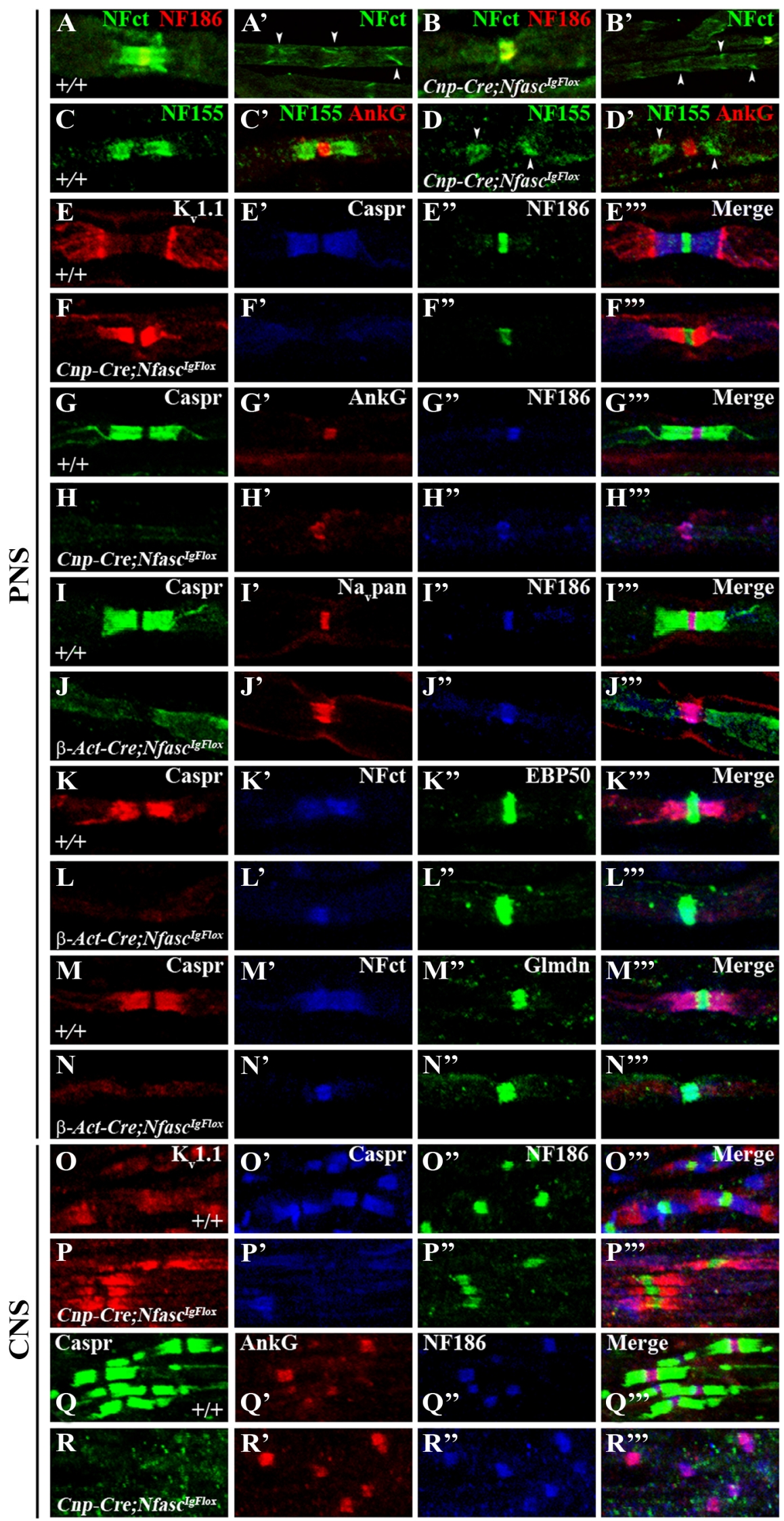

Figure 2. Loss of Ig5-6 in Nfasc ${ }^{\mathrm{NF} 155}$ results in axonal domain disorganization. $\boldsymbol{A}-\boldsymbol{R}$, Sciatic nerve fibers from $(\boldsymbol{A}-\boldsymbol{H}) \mathrm{P} 20$

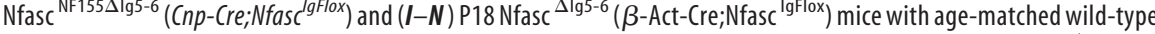
$(+/+)$ littermates and cervical spinal cord white matter tracts from $(\mathbf{O}-\boldsymbol{R})$ P20 wild-type $(+/+)$ and Nfasc ${ }^{\mathrm{NF} 155 \Delta \mathrm{Lg} 5-6}$ www.jneurosci.org as supplemental material). Overall, these results suggest that Ig5-6 are dispensable for Nfasc ${ }^{\mathrm{NF} 186}$ function in localizing, stabilizing, and organizing the node but are critical for $\mathrm{Nfasc}^{\mathrm{NF} 155}$ function in forming and maintaining the paranodes.

Paranodal septate junction formation and organization is disrupted in Nfasc ${ }^{N F 155 \Delta I g 5-6}$ mutant mice

Because of the analogous phenotypes observed between complete loss of Nfasc ${ }^{\mathrm{NF} 155}$ and Nfasc ${ }^{\mathrm{NF} 155 \Delta \mathrm{Ig} 5-6}$ at the light microscopic level, we used electron microscopic analysis to characterize the anatomical organization of myelinated fibers in $N$ fasc $^{N F 155 \Delta I g 5-6}$ mice (Fig. 3). Specific attention was focused on the septate-like junctions formed between paranodal loops and the axon. These junctions appear as ladder-like electron dense transverse bands in the paranodal space. Lowmagnification micrographs of wild-type $\left(+/+\right.$; Fig. 3A) and Cnp-Cre; Nfasc ${ }^{\text {IgFlox }}$ nerve fibers (Fig. 3B) appeared similar, except for the presence of organelles within the node(s) of Cnp-Cre;Nfasc IgFlox mice. This accumulation is consistent with previous findings observed in mice with disrupted paranodal axo-glial junctions (Einheber et al., 2006; Garcia-Fresco et al., 2006; Pillai et al., 2007). Closer examination revealed loss of septae in the paranodal region of Cnp-Cre; $\mathrm{Nfasc}^{\text {IgFlox }}$ nerves (Fig. $3 D$ ) compared to the wildtype fibers where the septae are clearly visible (Fig. 3C). Concomitant with the loss of septae, organelle accumulation was also observed in Cnp-Cre;Nfasc IgFlox nerves (Fig. 3F, arrows) compared with wildtype $(+/+$, Fig. $3 E)$. A similar loss of paranodal septae was observed in myelinated CNS spinal cord fibers in Cnp-Cre; Nfasc ${ }^{\text {IgFlox }}$ mice (Fig. 3H) compared to wild-type (Fig. 3G, arrowheads). The paranodal loops were often everted (EL) away from the axon and astrocytic processes (asterisks) infiltrated the paranodal space in the CNS of Cnp-Cre; $\mathrm{Nfasc}^{\text {IgFlox }}$ mice (Fig. $3 I, J)$. Moreover, the parallel arrays of cytoskeletal neurofilaments were disrupted in Cnp-Cre; Nfasc ${ }^{\text {IgFlox }}$ CNS fibers (Fig. 3L, arrowheads). Similarly, cross sections of cerebellar white matter from Cnp-Cre;

(Cnp-(re; Nfasd $\left.{ }^{\text {gFlox }}\right)$ mice were immunostained with a host of antibodies against juxtaparanodal, paranodal, and nodal proteins. All images were collected using identical confocal settings as the control and are representative of the three independent experiments. 

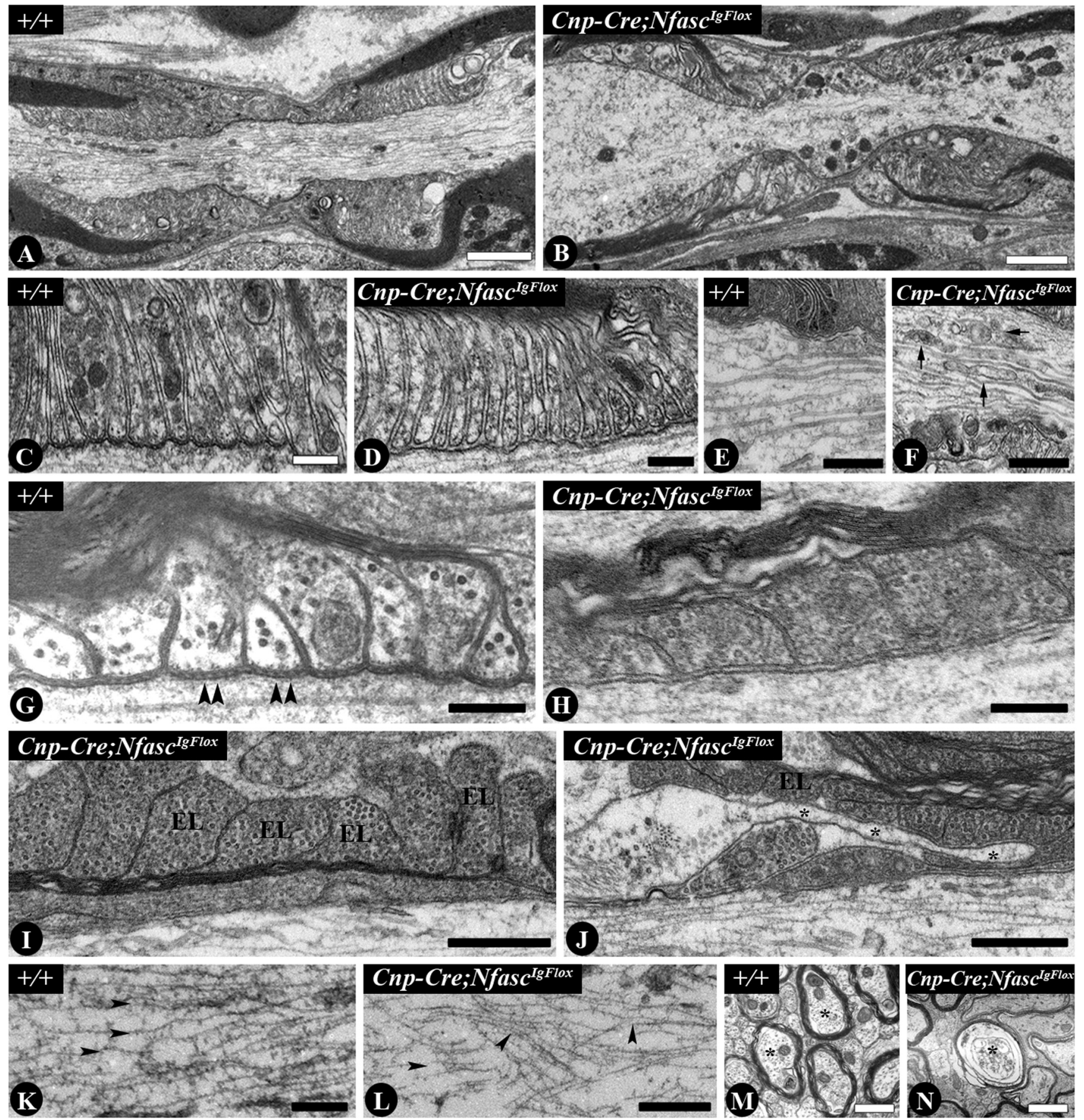

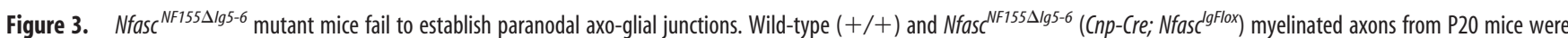
analyzed by electron microscopy. $\boldsymbol{A}-\boldsymbol{L}$, Longitudinal sections were taken of PNS sciatic nerve fibers $(\boldsymbol{A}-\boldsymbol{H})$ and spinal cord white matter tracts $(\boldsymbol{I}-\boldsymbol{L})$. $\boldsymbol{M}, \boldsymbol{N}$, (ross sections of Purkinje axons within the cerebellar white matter. Arrows in $\boldsymbol{F}$ denote organelle accumulation. Arrowheads in $\boldsymbol{G}$ highlight the electron-dense paranodal septate-like junctions. Arrowheads in $\boldsymbol{K}-\boldsymbol{L}$ mark the arrays of neurofilaments of the axonal cytoskeleton. Asterisks in $\boldsymbol{J}$ indicate the infiltrating astrocytic process, and in $\boldsymbol{M}-\boldsymbol{N}$ they mark axons of interest. EL indicates everted myelin loops. Scale bars represent $0.2 \mu \mathrm{m}(\boldsymbol{C}, \boldsymbol{D}, \mathbf{G}-\boldsymbol{J}), 0.5 \mu \mathrm{m}(\boldsymbol{E}, \boldsymbol{F}, \boldsymbol{K}, \boldsymbol{L})$, and $1 \mu \mathrm{m}(\boldsymbol{A}, \boldsymbol{B}, \boldsymbol{M}, \boldsymbol{N})$.

Nfasc ${ }^{I g F l o x}$ mice (Fig. $\left.3 N\right)$ revealed the presence of degenerating vacuoles within axons (asterisks), compared to wild-type (Fig. $3 M$, asterisks). We also observed the presence of swellings in Purkinje neuron axons of Cnp-Cre; Nfasc ${ }^{I g F l o x}$ mice similar to Cnp-Cre; Nfasc ${ }^{\text {Flox }}$ mice (supplemental Figure 3, available at www. jneurosci.org as supplemental material) (Pillai et al., 2009). Together, these results reveal an important role for Ig5-6 in the ability of $\mathrm{Nfasc}^{\mathrm{NF} 155}$ to coordinate and/or form paranodal septate junctions.
Ig5-6 are essential for the association of neurofascins with contactin, but not with gliomedin

Several reports have suggested a possible interaction between $\mathrm{Nfasc}^{\mathrm{NF155}}$ and Cont/Caspr within the paranodal domains of myelinated fibers, but the exact mechanisms governing these interactions remain unclear (Charles et al., 2002; Gollan et al., 2003; Bonnon et al., 2007). Previously, we reported that Nfasc ${ }^{\mathrm{NF} 155}$ preferentially bound a high-mannose, $\mathrm{N}$-glycosylated form of Cont in vitro (Bonnon et al., 2007). Here, we assessed whether 
A

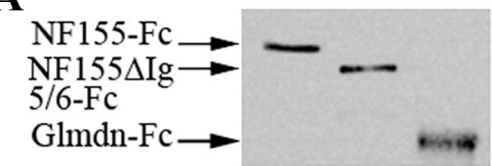

C
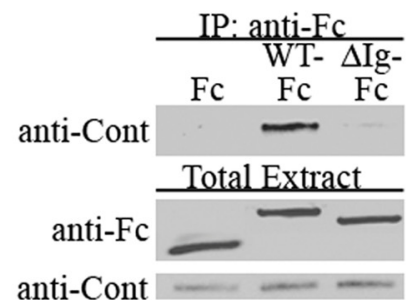

B

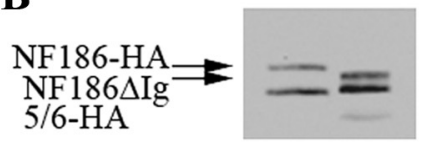

D

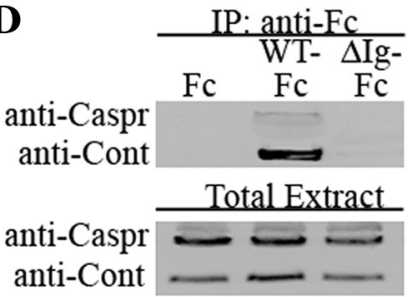

$\mathbf{E}$ $\mathrm{NF} 155-\mathrm{Fc}$
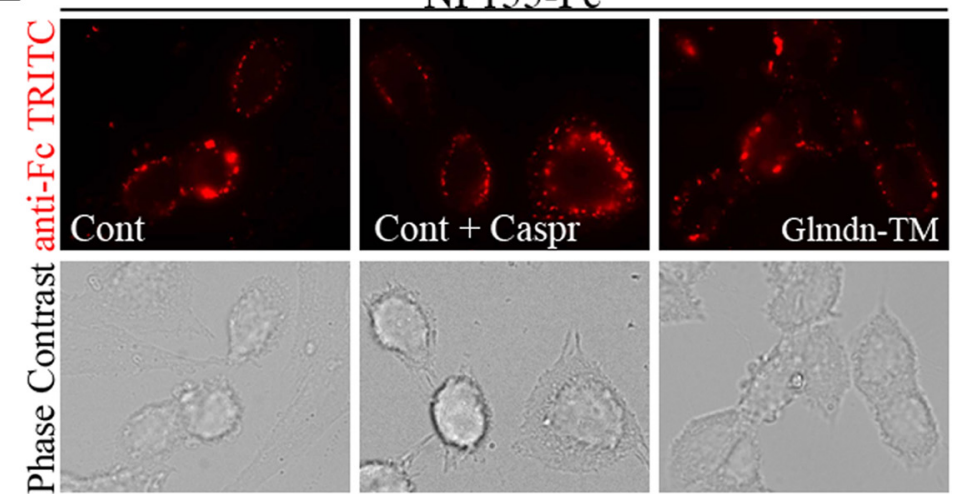

F

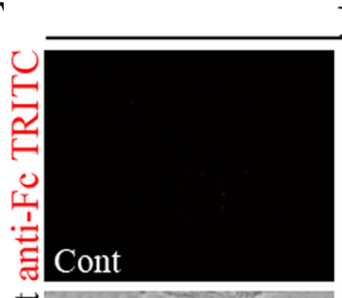

$\mathrm{NF} 155 \Delta \operatorname{Lg} 5 / 6-\mathrm{Fc}$
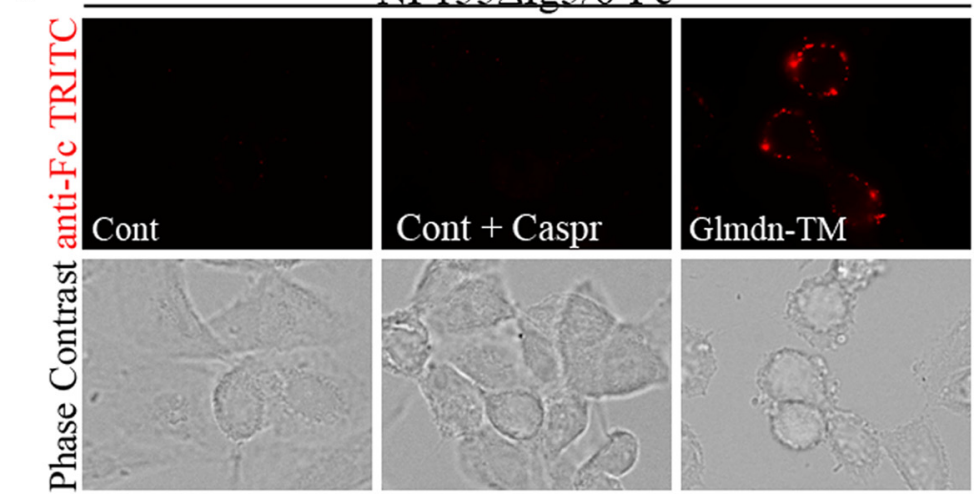

G
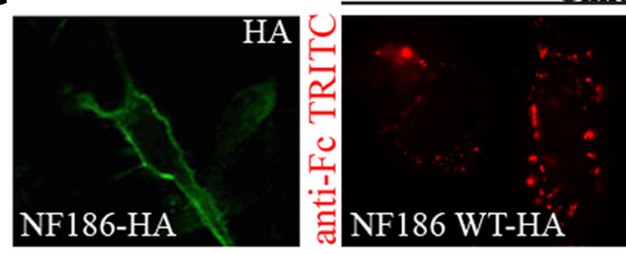

Glmdn-Fc
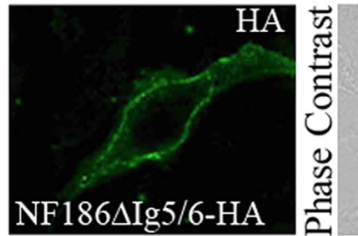

Figure 4. Ig $5-6$ are essential for Nfasc ${ }^{\mathrm{NF} 155}$ association with Cont. $A, B$, Immunoblot analysis of transiently transfected HEK293 (A) cells expressing Fc-tagged wild-type $\mathrm{Nfasc}^{\mathrm{NF} 155}$ (NF155-Fc), Nfasc ${ }^{\mathrm{NF} 155}$ lacking lg5-6 (NF155 $\Delta \mathrm{lg} 5 / 6-\mathrm{Fc}$ ), and Glmdn (Glmdn$\mathrm{Fc}$ ); and CHO-Lec1 (B) cells transiently expressing HA-tagged wild-type Nfasc ${ }^{\mathrm{NF} 186}$ (NF186-HA), and Nfasc ${ }^{\mathrm{NF} 186}$ deleted of Ig5-6 (NF186 $\Delta \mathrm{lg} 5 / 6-H A)$. C, D, In vitro pull-down assays of CHO-Lec1 cells transiently expressing Cont (C) or rat brain lysates (D) against Fc alone, wild-type Nfasc ${ }^{\mathrm{NF} 155}(\mathrm{WT}-\mathrm{Fc})$, and $\mathrm{lg} 5-6$ deleted Nfasc ${ }^{\mathrm{NF} 155}(\Delta \mathrm{lg}-\mathrm{Fc}) . \boldsymbol{E}, \boldsymbol{F}$, Immunobinding assays of CH0-Lec1 cells transiently expressing Cont alone, Cont with Caspr (Cont + Caspr), or Glmdn (Glmdn-TM) exposed to NF155-Fc (E), or NF155 $\Delta$ lg5/
Ig5-6 are critical for the association of $\mathrm{Nfasc}^{\mathrm{NF} 155}$ with Cont and/or Caspr, and Nfasc $^{\mathrm{NF} 186}$ with Glmdn (Fig. 4). First, Fctagged constructs of wild-type Nfasc ${ }^{\mathrm{NF} 155}$ (NF155-Fc), Nfasc ${ }^{\mathrm{NF} 155}$ with deleted Ig5-6 (NF155 $\Delta$ Ig5/6-Fc), and Glmdn (Glmdn$\mathrm{Fc}$ ), and HA-tagged constructs of wild-type Nfasc $^{\text {NF186 }}$ (NF186-HA) and Nfasc ${ }^{\text {NF186 }}$ lacking Ig5-6 (NF186 $\Delta$ Ig5/6-HA) were produced and expressed in HEK-293 (Fig. $4 A$ ) and CHO-Lec1 cells (Fig. $4 B$ ), respectively. The deletion of Ig domains 5 and 6 encompassed amino acids 414 to 597 of Nfasc and was identical to the targeting construct used to develop the $N$ fasc $^{\text {IgFlox }}$ mice. Next, we performed pulldown assays using the Fc-tagged Nfasc ${ }^{\mathrm{NF} 155}$ proteins against lysates from $\mathrm{CHO}-\mathrm{Lec} 1$ cells overexpressing Cont (Fig. 4C), or rat brain lysates (Fig. 4D) endogenously expressing Cont and Caspr. Whereas wildtype Nfasc ${ }^{\mathrm{NF} 155}$ (WT-FC) precipitated Cont and Cont+Caspr from CHO-Lec1 cell and rat brain lysates, respectively, the ability of Nfasc ${ }^{\mathrm{NF} 155}$ lacking Ig5-6 ( $\Delta \mathrm{Ig}$ Fc) to precipitate Cont and/or Caspr from cell lysates was dramatically reduced. Total extracts from cell lysates showed that equal levels of Cont, Caspr, and the Fctagged proteins were expressed, therefore, these findings indicate that Ig5-6 are essential for the interaction of Nfasc ${ }^{\mathrm{NF} 155}$ with Cont.

Next we performed in vitro binding assays where CHO-Lec1 cells transiently expressing Cont, Cont+Caspr, or Glmdn were exposed to the different Fc-Tagged Nfasc ${ }^{\text {NF155 }}$ proteins. Similar to the pulldown assays, NF155 $\mathrm{Ig} 5 / 6-\mathrm{Fc}$ was not able to stably associate with $\mathrm{CHO}-\mathrm{Lec} 1$ cells expressing Cont, or Cont + Caspr (Fig. $4 F)$, compared to wild-type $\mathrm{Nfasc}^{\mathrm{NF} 155}$ (NF155-Fc; Fig. 4E). Interestingly, loss of Ig5-6 from Nfasc ${ }^{\mathrm{NF} 155}$ did not affect its ability to bind Glmdn. This result is of no major consequence, as to date no functional relationship between these two proteins has been characterized. However, Glmdn can interact with $\mathrm{Nfasc}^{\mathrm{NF} 186}$ in vitro and is proposed to act in facilitating nodal organization in the PNS (Eshed et al., 2005). Therefore, we sought to determine whether Ig5-6 of Nfasc ${ }^{\mathrm{NF} 186}$ are critical for its interaction with Glmdn in vitro. HA-tagged Nfasc ${ }^{\mathrm{NF} 186}$ (NF186 WTHA) and Nfasc ${ }^{\text {NF186 }}$ deleted for Ig5-6

6-FC (F). G, Immunostaining of CHO-Lec1 cells transiently expressing NF186-HA and NF186 $\mathrm{lg} 5 / 6-\mathrm{HA}$ and binding assays GImdn-Fc. Phase contrast images of the transfected cells (in $\boldsymbol{E}$, $\boldsymbol{F}, \boldsymbol{G})$ indicate the health, numbers, and viability of the cells. 
(NF186 $\mathrm{Ig} 5 / 6-\mathrm{HA})$ both localized to the plasma membranes of transiently expressing $\mathrm{CHO}-\mathrm{Lec} 1$ cells (Fig. $4 G$ ). When exposed to Fc-tagged Glmdn, both NF186-HA and NF186 Ig5-6-HA transiently expressing CHO-Lec1 cells bound Glmdn (Fig. 4G). These data are consistent with the observation that loss of Ig5-6 in Nfasc ${ }^{\mathrm{NF} 186}$ did not affect the recruitment or expression of Glmdn to PNS nodes (Fig. $2 \mathrm{~N}^{\prime \prime}$ ). Taken together, these results demonstrate that Ig5-6 are indispensable for the stable association of $\mathrm{Nfasc}^{\mathrm{NF} 155}$ with the paranodal component Cont and is the first mechanistic evidence identifying critical domains within Nfasc that are required for its proper functioning in establishing the paranodal axo-glial junctions.

\section{Discussion}

Recent findings have advanced our knowledge of key molecules regulating myelination, and more specifically axonal domain organization, but little is known of how these domains become organized during nervous system development. Here, using mutational analysis, we find that Ig5-6 of Nfasc ${ }^{\mathrm{NF155}}$, but not Nfasc ${ }^{\mathrm{NF} 186}$, are essential for axonal domain organization and integrity by facilitating the stable association of $\mathrm{Nfasc}^{\mathrm{NF} 155}$ with Cont, both in vivo and in vitro. These studies are the first to reveal domain-specific requirements for paranodal axon-glial interactions and therefore may provide valuable clues to the mechanisms leading to disorganization of axonal domains during disease onset and progression.

\section{Ig domain perturbation resembles complete ablation of Nfasc $^{\mathrm{NF155}}$}

Mice lacking Nfasc ${ }^{\mathrm{NF} 155}$ expression presented with severe motor coordination defects (Pillai et al., 2009) similar to Caspr and Cont mutant mice and survived to postnatal day 17 (Bhat et al., 2001; Boyle et al., 2001). Here, we find that loss of Ig5-6 alone from $\mathrm{Nfasc}^{\mathrm{NF} 155}$ in mice completely recapitulates the phenotypes of $N$ fasc $^{N F 155}$ null mice, including the onset of paralysis and ataxia, paranodal disorganization, and loss of paranodal septae. These findings suggest that Ig5-6 may act to coordinate Cont or Cont/ Caspr localization to the paranodes during myelination. When closely examined, we found that Cnp-Cre; Nfasc ${ }^{\text {IgFlox }}$ myelinated nerves expressed the mutant Nfasc ${ }^{\mathrm{NF} 155 \Delta \mathrm{Ig} 5-6}$ protein within the Schmidt-Lantermann incisures (Fig. $2 B$ ) and the paranodes (Fig. $2 D$ ) demonstrating that loss of Ig5-6 does not perturb the ability of Nfasc ${ }^{\mathrm{NF} 155}$ to localize to the paranodal plasma membrane. Therefore, it is likely that these domains are responsible for promoting the association of $\mathrm{Nfasc}^{\mathrm{NF} 155}$ with Cont and/or Caspr, thereby stabilizing the paranodal complex. Together, these results show that Ig5-6 are critical for the stabilization of the paranodal axo-glial junctions.

\section{Association of $\mathrm{Nfasc}^{\mathrm{NF155}}$ with Cont/Caspr complex is mediated by Ig5-6}

The analogous paranodal deformities observed between $N f a s c^{N F 155}$, Nfasc ${ }^{N F 155 \Delta I g 5-6}$, Caspr, and Cont mutant mice supports the hypothesis that these proteins may interact in a complex to facilitate axonal domain organization in myelinated fibers as is observed in invertebrate septate junction formation (Faivre-Sarrailh et al., 2004; Banerjee and Bhat, 2008). Initial vertebrate studies performed in vitro demonstrated that the extracellular domain of Nfasc ${ }^{\mathrm{NF} 155}$ was capable of associating with the Cont/Caspr complex (Charles et al., 2002). Yet another study suggested that Caspr perturbed the association of Cont and $\mathrm{Nfasc}^{\mathrm{NF155}}$ in vitro, thus the true mechanism of these interactions remains to be established (Gollan et al., 2003). To address this issue, we previously showed, in vitro, that $\mathrm{Nfasc}^{\mathrm{NF} 155}$ associated with a glycoslated form of Cont in the presence or absence of Caspr (Bonnon et al., 2007). Here, we find that Ig5-6 are critical for the interaction of $\mathrm{Nfasc}^{\mathrm{NF} 155}$ with Cont. In vitro pull-down assays revealed a dramatic reduction in the ability of $\mathrm{Nfasc}^{\mathrm{NF} 155}$ to associate with Cont, or Cont +Caspr, in the absence of Ig5-6 (Fig. 4C,D). Accordingly, cell binding assays indicated that Fc-tagged Nfasc ${ }^{\mathrm{NF} 155}$ lacking Ig5-6 was not able to stably adhere to cells overexpressing Cont or Cont + Caspr and thus coincide with our in vivo data showing diffuse distribution of Caspr and Cont in Nfasc ${ }^{\mathrm{NF} 155 \Delta \mathrm{Ig} 5-6}$ myelinated axons. Interestingly, $\mathrm{Nfasc}^{\mathrm{NF} 155}$ was able to associate with Glmdn regardless of the presence of Ig5-6, indicating that ablation of these domains does not significantly alter the protein structure or protein folding of Nfasc ${ }^{\mathrm{NF} 155}$. Overall, these results provide evidence supporting a role for $\mathrm{Nfasc}^{\mathrm{NF} 155}$ in coordinating paranodal axo-glial junction formation, organization, and stabilization by binding Cont and facilitating complex formation with Caspr.

\section{Domain-specific requirements for the function of neurofascins during nervous system development}

To date, up to fifty alternatively spliced variants of the Nfasc gene have been identified in chicken, but only two major murine isoforms have been characterized as critical regulators of nervous system development, glial-specific $\mathrm{Nfasc}^{\mathrm{NF} 155}$ and neuronalspecific Nfasc $^{\text {NF186 }}$ (Koticha et al., 2006; Hassel et al., 1997; Davis et al., 1996). Because of the high percentage of homology in the extracellular domains of $\mathrm{Nfasc}^{\mathrm{NF155}}$ and $\mathrm{Nfasc}^{\mathrm{NF} 186}$, it is plausible that they function equivalently to organize and stabilize their paranodes and nodes, respectively. By using $\beta$-Actin-Cre, we ablated Ig5-6 from Nfasc ${ }^{\mathrm{NF} 155}$ and Nfasc ${ }^{\mathrm{NF} 186}$. Concomitant loss of Ig5-6 from both isoforms led to paranodal disruption identical to that observed in mice deficient for Ig5-6 in Nfasc ${ }^{\mathrm{NF} 155}$ alone. Interestingly, ablation of Ig5-6 from Nfasc ${ }^{\mathrm{NF} 186}$ did not result in nodal disorganization, as Nfasc ${ }^{\mathrm{NF} 186}$, along with several other major nodal components localized to nodes, even in the absence of intact paranodes (Fig. 2). Furthermore, electrophysiological data revealed no change in conduction velocity between Cnp-Cre; $N f a s c^{\text {IgFlox }}$ mice and $\beta$-Act-Cre;Nfasc ${ }^{\text {IgFlox }}$ mice (supplemental Figure 4, available at www.jneurosci.org as supplemental material). If, indeed, the Ig domains were essential for Nfasc ${ }^{\mathrm{NF} 186}$ function, one would have expected a more profound or cumulative decline in nerve conduction velocity in $\beta$-Act-Cre; Nfasc IfFlox mice compared to Cnp-Cre; Nfasc ${ }^{\text {IgFlox }}$ mice. Additionally, the in vitro binding assays revealed that $\mathrm{Nfasc} \mathrm{NF}^{\mathrm{N} 86}$ maintained its ability to associate with Glmdn in the absence of Ig5-6. Taken together, these results demonstrate that individual isoforms of Nfasc have distinct domain requirements regulating their ability to interact with and organize regional-specific components within myelinated axons.

In summary, we have provided evidence of specific domains within $\mathrm{Nfasc}^{\mathrm{NF155}}$ that are critical for paranodal formation and overall axonal domain organization and integrity. Perturbation of these domains resulted in severe paranodal abnormalities, disorganized axonal domains, and significant nerve conduction delays. Future in vivo structure/function studies, aimed at identifying critical regions of protein-protein interactions between key molecular players coordinating domain organization, will be invaluable in elucidating the mechanisms underlying neuronglia interactions in myelinated axons.

\section{References}

Banerjee S, Bhat MA (2008) Glial ensheathment of peripheral axons in Drosophila. J Neurosci Res 86:1189-1198.

Bhat MA, Rios JC, Lu Y, Garcia-Fresco GP, Ching W, St Martin M, Li J, 
Einheber S, Chesler M, Rosenbluth J, Salzer JL, Bellen HJ (2001) Axonglia interactions and the domain organization of myelinated axons requires neurexin IV/Caspr/Paranodin. Neuron 30:369-383.

Bonnon C, Bel C, Goutebroze L, Maigret B, Girault JA, Faivre-Sarrailh C (2007) PGY repeats and N-glycans govern the trafficking of paranodin and its selective association with contactin and neurofascin-155. Mol Biol Cell 18:229-241.

Boyle ME, Berglund EO, Murai KK, Weber L, Peles E, Ranscht B (2001) Contactin orchestrates assembly of the septate-like junctions at the paranode in myelinated peripheral nerve. Neuron 30:385-397.

Charles P, Tait S, Faivre-Sarrailh C, Barbin G, Gunn-Moore F, DenisenkoNehrbass N, Guennoc AM, Girault JA, Brophy PJ, Lubetzki C (2002) Neurofascin is a glial receptor for the paranodin/Caspr-contactin axonal complex at the axoglial junction. Curr Biol 12:217-220.

Davis JQ, Lambert S, Bennett V (1996) Molecular composition of the node of Ranvier: identification of ankyrin-binding cell adhesion molecules neurofascin (mucin+/third FNIII domain-) and NrCAM at nodal axon segments. J Cell Biol 135:1355-1367.

Durbec P, Gennarini G, Buttiglione M, Gomez S, Rougon G (1994) Different domains of the F3 neuronal adhesion molecule are involved in adhesion and neurite outgrowth promotion. Eur J Neurosci 6:461-472.

Einheber S, Bhat MA, Salzer JL (2006) Disrupted axo-glial junctions result in accumulation of abnormal mitochondria at nodes of Ranvier. Neuron Glia Biol 2:165-174.

Eshed Y, Feinberg K, Poliak S, Sabanay H, Sarig-Nadir O, Spiegel I, Bermingham JR Jr, Peles E (2005) Gliomedin mediates Schwann cell-axon interaction and the molecular assembly of the nodes of Ranvier. Neuron 47:215-229.

Faivre-Sarrailh C, Banerjee S, Li J, Hortsch M, Laval M, Bhat MA (2004) Drosophila contactin, a homolog of vertebrate contactin, is required for septate junction organization and paracellular barrier function. Development 131:4931-4942.

Garcia-Fresco GP, Sousa AD, Pillai AM, Moy SS, Crawley JN, Tessarollo L, Dupree JL, Bhat MA (2006) Disruption of axo-glial junctions causes cytoskeletal disorganization and degeneration of Purkinje neuron axons. Proc Natl Acad Sci U S A 103:5137-5142.

Gollan L, Salomon D, Salzer JL, Peles E (2003) Caspr regulates the processing of contactin and inhibits its binding to neurofascin. J Cell Biol 163:1213-1218.

Hassel B, Rathjen FG, Volkmer H (1997) Organization of the neurofascin gene and analysis of developmentally regulated alternative splicing. J Biol Chem 272:28742-28749.

Koticha D, Maurel P, Zanazzi G, Kane-Goldsmith N, Basak S, Babiarz J, Salzer J, Grumet M (2006) Neurofascin interactions play a critical role in clus- tering sodium channels, ankyrin G and beta IV spectrin at peripheral nodes of Ranvier. Dev Biol 293:1-12.

Lappe-Siefke C, Goebbels S, Gravel M, Nicksch E, Lee J, Braun PE, Griffiths IR, Nave KA (2003) Disruption of Cnp1 uncouples oligodendroglial functions in axonal support and myelination. Nat Genet 33:366-374.

Lonigro A, Devaux JJ (2009) Disruption of neurofascin and gliomedin at nodes of Ranvier precedes demyelination in experimental allergic neuritis. Brain 132:260-273.

Melendez-Vasquez CV, Rios JC, Zanazzi G, Lambert S, Bretscher A, Salzer JL (2001) Nodes of Ranvier form in association with ezrin-radixin-moesin (ERM)-positive Schwann cell processes. Proc Natl Acad Sci U S A 98:1235-1240.

Menegoz M, Gaspar P, Le Bert M, Galvez T, Burgaya F, Palfrey C, Ezan P, Arnos F, Girault JA (1997) Paranodin, a glycoprotein of neuronal paranodal membranes. Neuron 19:319-331.

Pillai AM, Garcia-Fresco GP, Sousa AD, Dupree JL, Philpot BD, Bhat MA (2007) No effect of genetic deletion of contactin-associated protein (CASPR) on axonal orientation and synaptic plasticity. J Neurosci Res 85:2318-2331.

Pillai AM, Thaxton C, Pribisko AL, Cheng JG, Dupree JL, Bhat MA (2009) Spatiotemporal ablation of myelinating glia-specific neurofascin (Nfasc NF155) in mice reveals gradual loss of paranodal axoglial junctions and concomitant disorganization of axonal domains. J Neurosci Res 87:1773-1793

Rigato F, Garwood J, Calco V, Heck N, Faivre-Sarrailh C, Faissner A (2002) Tenascin-C promotes neurite outgrowth of embryonic hippocampal neurons through the alternatively spliced fibronectin type III BD domains via activation of the cell adhesion molecule F3/contactin. J Neurosci 22:6596-6609.

Salzer JL (2003) Polarized domains of myelinated axons. Neuron 40: 297-318.

Sherman DL, Tait S, Melrose S, Johnson R, Zonta B, Court FA, Macklin WB Meek S, Smith AJ, Cottrell DF, Brophy PJ (2005) Neurofascins are required to establish axonal domains for saltatory conduction. Neuron 48:737-742.

Stanley P, Ioffe E (1995) Glycosyltransferase mutants: key to new insights in glycobiology. FASEB J 9:1436-1444.

Thaxton C, Bhat MA (2009) Myelination and regional domain differentiation of the axon. Results Probl Cell Differ 48:1-28.

Zonta B, Tait S, Melrose S, Anderson H, Harroch S, Higginson J, Sherman DL, Brophy PJ (2008) Glial and neuronal isoforms of Neurofascin have distinct roles in the assembly of nodes of Ranvier in the central nervous system. J Cell Biol 181:1169-1177. 